\title{
Genetic variation of Echinococcus spp. in yaks and sheep in the Tibet Autonomous Region of China based on mitochondrial DNA
}

\author{
John Asekhaen Ohiolei ${ }^{1 \dagger}$, Chen-Yang Xia ${ }^{2 \dagger}$, Li Li ${ }^{1}$, Jian-Zhi Liu² ${ }^{2}$ Wen-Qiang Tang ${ }^{2}$, Yan-Tao Wu', Danqulamu², \\ Guo-Qiang Zhu' ${ }^{1}$, Bin Shi ${ }^{2}$, Bao-Quan Fu' ${ }^{1}$, Hong Yin ${ }^{1}$, Hong-Bin Yan ${ }^{1 *}$ and Wan-Zhong Jia ${ }^{\text {** }}$
}

\begin{abstract}
Background: Cystic echinococcosis (CE) in humans and livestock is caused by Echinococcus granulosus (sensu lato). In China where CE is endemic, a number of studies have shown that Echinococcus granulosus (sensu stricto) is majorly responsible for $\mathrm{CE}$. However, E. canadensis (G6) which is the second leading cause of CE is now being detected in most parts of the country. In this study, the species diversity and genetic variation of Echinococcus granulosus (s.l.) in four counties in Tibet Autonomous Region of China were investigated.
\end{abstract}

Methods: Infection with Echinococcus granulosus (s.s.) in yaks and sheep was identified using NADH dehydrogenase subunit 1 and 5 (nad 1 and nad5) mitochondrial genes while the genotype G6 of E. canadensis initially diagnosed with $\mathrm{NADH}$ dehydrogenase subunit 1 (nad1) was further confirmed by analysis of the complete mitochondrial genome and a phylogenetic network constructed based on the nad 2 and nad5 genes.

Results: Out of 85 hydatid cyst samples collected from slaughtered sheep $(n=54)$ and yaks $(n=31)$, 83 were identified as E. granulosus (s.s.) G1 ( $n=77), G 3(n=6)$ and 2 were identified as E. canadensis G6. Analysis of the nad1/nad5 genes revealed 16/17 mutations with 9/14 parsimony informative sites resulting in 15/14 haplotypes, respectively. Haplotype diversity $(\mathrm{Hd})$ and nucleotide diversity $(\pi)$ of E. granulosus (s.s.) population were 0.650 and 0.00127 for nad 1 and 0.782 and 0.00306 for nad5, respectively, with an overall negative Tajima's $D$ and Fu's Fs. A low $F_{\text {ST }}$ indicated no genetic difference between isolates from sheep and yaks.

Conclusion: Pockets of infection with E. canadensis (G6, G7, G8 and G10) have been previously reported in sheep, goats, yaks and/or humans in different parts of China. While the G6 genotype has been previously reported in sheep in the Tibet Autonomous Region, the detection in a yak in the present study represents the first to the best of our knowledge. Therefore, we recommend future surveys and control efforts to comprehensively investigate other potential intermediate hosts for the prevalence and genetic diversity of the E. canadensis group (G6, G7, G8 and G10) across the country and their inclusion into the existing CE control programme.

Keywords: Echinococcus canadensis, Haplotypes, Genetic variation, Tibet Autonomous Region

\footnotetext{
*Correspondence: yanhongbin@caas.cn; jiawanzhong@caas.cn

† John Asekhaen Ohiolei and Chen-Yang Xia contributed equally to this manuscript

${ }^{1}$ State Key Laboratory of Veterinary Etiological Biology/National Professional Laboratory of Animal Hydatidosis, Key Laboratory of Veterinary Parasitology of Gansu Province/Lanzhou Veterinary Research Institute, CAAS, Lanzhou 730046, Gansu, People's Republic of China

Full list of author information is available at the end of the article
}

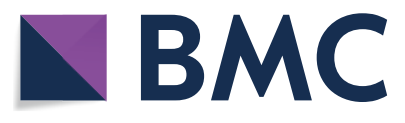

(c) The Author(s) 2019. This article is licensed under a Creative Commons Attribution 4.0 International License, which permits use, sharing, adaptation, distribution and reproduction in any medium or format, as long as you give appropriate credit to the original author(s) and the source, provide a link to the Creative Commons licence, and indicate if changes were made. The images or other third party material in this article are included in the article's Creative Commons licence, unless indicated otherwise in a credit line to the material. If material is not included in the article's Creative Commons licence and your intended use is not permitted by statutory regulation or exceeds the permitted use, you will need to obtain permission directly from the copyright holder. To view a copy of this licence, visit http://creativeco mmons.org/licenses/by/4.0/. The Creative Commons Public Domain Dedication waiver (http://creativecommons.org/publicdomain/ zero/1.0/) applies to the data made available in this article, unless otherwise stated in a credit line to the data. 


\section{Background}

Cystic echinococcosis (CE) is a parasitic zoonosis caused by tapeworm species of Echinococcus granulosus (s.l.), which infect humans and livestock upon ingestion of the infective egg stage from the definitive host resulting in the development of hydatid cyst of the metacestode larval stages in the liver, lungs and sometimes in other organs. Within the E. granulosus complex, high genetic variation which affects species/genotype infectivity and preference to intermediate hosts has been recognised based on studies of parasite mitochondrial DNA [1-3]. So far, known species in this complex include E. granulosus (sensu stricto) (G1/G3), E. equinus (G4), E. ortleppi (G5), E. canadensis (G6, G7, G8 and G10) and E. felidis [4]. Meanwhile, the species status of the $E$. canadensis group is still under debate as suggestions have been made to reclassify the group into E. intermedius (G6/7), E. borealis (G8) and E. canadensis (G10) $[5,6]$. Nonetheless, for the purpose of convenience, we refer to the group as E. canadensis all through the report.

CE poses a serious public health concern in China as a result of livestock infection, increasing morbidity in humans and the consequent economic impact due to the cost of diagnosis, control and treatment. The prevalence of CE in China varies with location with the Qinghai-Tibet Plateau being the most endemic region. In China, a number of molecular epidemiological studies have identified E. granulosus (s.s.) as the leading cause of cystic echinococcosis [7-12]. However, pockets of infection due to E. canadensis (G6, G7, G8 and G10) are now being reported [12-15]. Previously, infection with E. canadensis G6 genotype was frequently reported in the Xinjiang Uygur Autonomous Region [16, 17]. However, recent reports of the G6 genotype and other members of E. canadensis group in areas other than Xinjiang Uygur Autonomous Region, are raising concerns on its host range and public health significance $[12,14,15,18]$. Therefore, it becomes imperative to update the status of $\mathrm{CE}$ and the genetic diversity of species especially in counties or communities in endemic regions, which will assist in monitoring the progress of ongoing CE management and control programmes across the country. In this study, the species diversity and genetic variation of Echinococcus species in four counties in the Tibet Autonomous Region of China were investigated and for the first time, the presence of $E$. canadensis (G6) in yak was confirmed using mitochondrial DNA markers (nad2 and nad5) and the complete mitochondrial genome analysis.

\section{Methods}

\section{Study area}

The Tibet Autonomous Region is a plateau with grassland vegetation. It has an average elevation of about
$4000 \mathrm{~m}$ and covers a landmass of about 1.23 million $\mathrm{km}^{2}$ bordering Qinghai Province and Xinjiang Uygur Autonomous Region to the north, Sichuan and Yunnan provinces to the east, and to the south, Myanmar, India, Bhutan and Nepal. Tibetans live mostly as seminomadic pastoralists with goats, sheep, yaks, horses and dogs as their main domestic animals. The Tibet region has a total of 74 counties in 7 prefectures that include 692 townships and 5260 villages with a total population of 3 million [19]. The study was carried out in four counties in the region namely: Angren, Saga and Zhongba (Shigatse Prefecture), and Dangxiong (Lhasa City) (Fig. 1).

\section{Sample collection and DNA extraction}

Between October and December 2017, a total of 85 hydatid cysts were collected from the lungs and liver of slaughtered sheep (age 1-11 years) and yaks (age 5-18 years) during routine meat inspection in slaughterhouses and slabs in the counties. The slaughtered animals were raised in an extensive management system (free grazing) in the same counties where the study was conducted. The collected hydatid cyst samples (Additional file 1: Figure S1, Additional file 2: Figure S2) were then cleaned with $75 \%$ alcohol before the germinal layers were removed and washed repeatedly with phosphate buffer solution (PBS) and then transported in an ice box to the laboratory where they were stored at $-80{ }^{\circ} \mathrm{C}$ prior to DNA extraction. Further, a piece of the germinal layer excised from each hydatid cyst was crushed in liquid nitrogen before genomic DNA extraction was carried out using Qiagen Blood and Tissue DNA Extraction Kit (Qiagen, Hilden, Germany) according to the manufacturer's instructions.

\section{DNA amplification and sequencing}

PCR was performed in a $25 \mu \mathrm{l}$ final reaction mixture containing $12.5 \mu$ l Premix $\operatorname{Taq}^{\text {TM }}$ (Takara Bio, Kusatsu, Japan), $0.5 \mu \mathrm{l}$ of each primer (10 pmol), $0.5 \mu \mathrm{l}$ of the genomic DNA (c. 10-180 ng), and $11 \mu \mathrm{l}$ RNAse-free water. The PCR conditions were as follows: initial denaturation at $95{ }^{\circ} \mathrm{C}$ for $5 \mathrm{~min} ; 35$ cycles of denaturation at $95^{\circ} \mathrm{C}$ for 30 $\mathrm{s}$, annealing at $55^{\circ} \mathrm{C}$ for $40 \mathrm{~s}$ and elongation at $72{ }^{\circ} \mathrm{C}$ for $60-90 \mathrm{~s}$; and a final extension step at $72{ }^{\circ} \mathrm{C}$ for $10 \mathrm{~min}$.

The complete mitochondrial NADH dehydrogenase subunit 1 gene (nad $1,894 \mathrm{bp}$ ) was initially amplified for all isolates using the forward (5'-ATT ATA GAA AAT TTT CGT TTT ACA CGC-3) and reverse (5'ATT CAC AAT TTA CTA TAT CAA AGT AAC C-3) primers [15]. In order to distinguish between genotypes of E. granulosus (s.s.) a primer pair amplifying a fragment of the nad5 gene (about $680 \mathrm{bp}$ ) reported to have conserved regions capable of discriminating G1 from G3 [20] was used, while the complete mitochondrial genome was amplified to correctly place the G6 
genotype using previously designed primers [15] and newly designed complementary primers (Additional file 3: Table S1). Each PCR reaction yielded a single band detected in a $1.5 \%(\mathrm{w} / \mathrm{v})$ agarose gel stained with GelRed $^{\circledR}$ (Biotium, Fremont, USA). Five microliters of the amplicon were used for visualization while the rest were used for sequencing in an ABI3730XI DNA Analyser (Tsinqke Biological Technology, Beijing, China).

\section{Molecular and phylogenetic analysis}

Following sequencing, the nucleotide sequences were viewed and manually corrected for any misread nucleotide by comparing the chromatogram file with the resulting sequences using Unipro UGENE v1.29.0 software and BioEdit [21]. The sequences of the complete mitochondrial genome were assembled stepwise with the help of DNAStar v7.1 program, BioEdit, and Unipro UGENE v1.29.0 software while making sure that the overlap sequences were identical. The final sequences were aligned with BioEdit [21]. The proteinencoding genes and rRNAs of the complete mitochondrial genome were annotated using GeSeq [22]; tRNAs were identified using tRNAscan-SE v2.0.3 and/or by a BLAST search against an E. canadensis genotype G6 mitochondrial genome sequence available in GenBank (GenBank: AB208063). The identity of the isolates via their nucleotide sequences was assessed using the NCBI
BLAST program and compared with existing sequences in the GenBank database (https://blast.ncbi.nlm.nih. gov/Blast.cgi).

The population diversity indices were estimated in DnaSP v6 [23]. Median-joining networks for E. granulosus (s.s.) and E. canadensis G6 were constructed based on the nucleotide sequences of the mitochondrial nad5 [20] and concatenated nad2-nad5 [24] genes, respectively using PopART software [25]. Using DnaSP v6 [23], the population neutrality indices, Tajima's D [26] and Fu's Fs [27], and pairwise fixation index $\left(\mathrm{F}_{\mathrm{ST}}\right)$, were calculated. A Bayesian phylogenetic tree for E. canadensis G6 genotype was infered based on the concatenation of the 12 protein coding genes using MrBayes v.3.1.2 with a Markov Chain Monte Carlo (MCMC) sampling to assess the posterior distribution of parameters using a chain length of 4,000,000 states with $10 \%$ discarded as 'burn-in'. The parameters were logged every 1000 states. Convergence was assessed by ensuring that the potential scale reduction factor (PSRF) approaches 1 [28] while the resulting tree was displayed by TreeView (http://taxonomy.zoolo gy.gla.ac.uk/rod/treeview.html).

\section{Results}

Out of the 85 isolates (54 from sheep and 31 from yaks) (Table 1), 83 were identified as E. granulosus (s.s.) comprising genotypes G1 $(n=77)$ and G3 $(n=6)$ and 2 (from a yak and sheep) were identified as $E$.

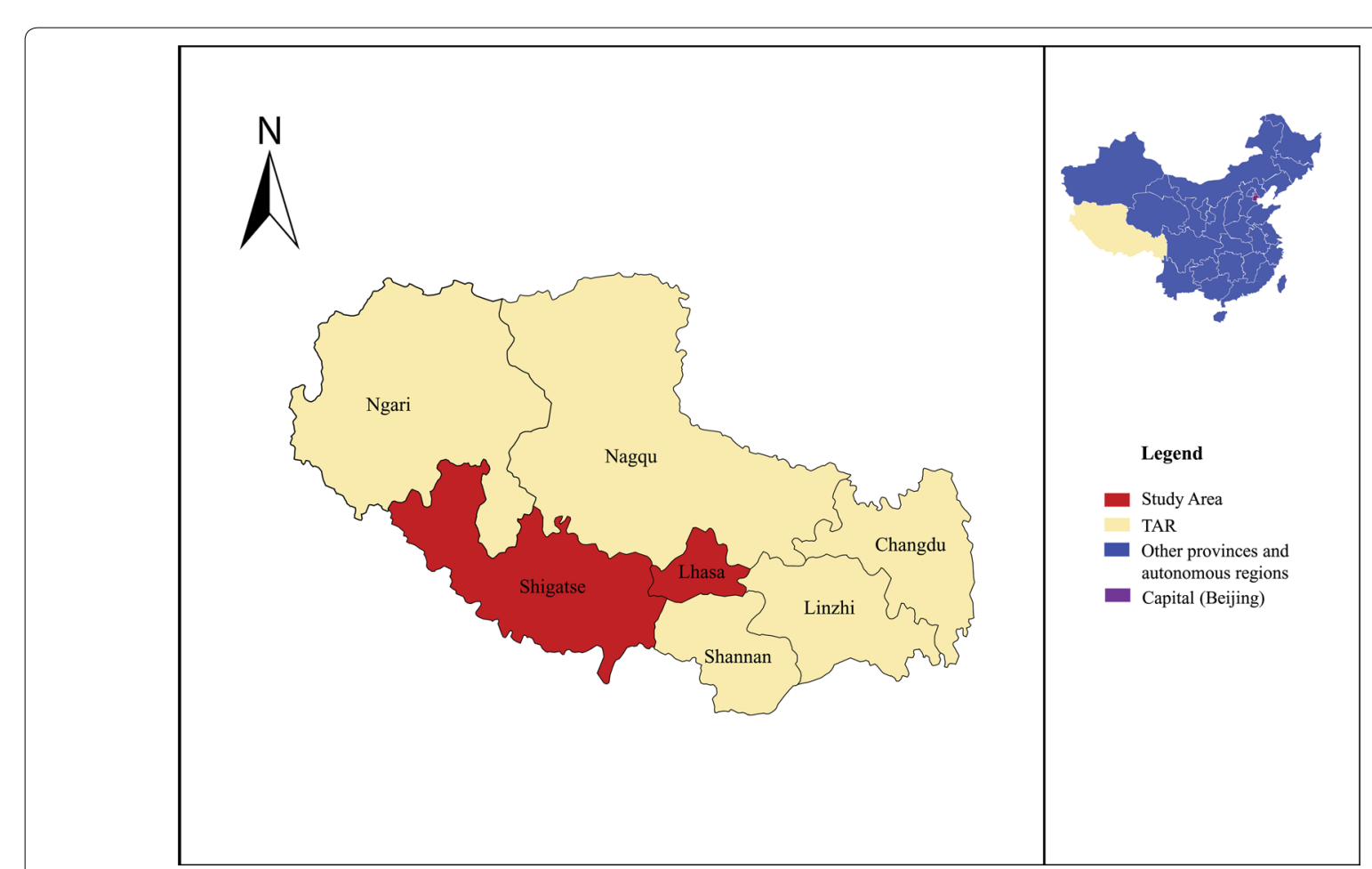

Fig. 1 Map of the Tibet Autonomous Region of China showing the study area 
canadensis (genotype G6) with > 99\% identity to deposited sequences in GenBank. The E. granulosus (s.s.) nad 1 gene sequences had a total of 16 polymorphic sites with 9 parsimony informative sites, while nad5 had a total of 17 mutations with 14 parsimony informative sites without indels. The observed nucleotide mutations also resulted in amino acid changes. Based on the nad5 (680 bp) gene, amino acid substitution between genotype G1 and G3 were observed at three positions as follows: $97, \mathrm{~S}$ to $\mathrm{T}$; 105, I to V; and 219, G to S (Additional file 3: Tables S2, S3).

\section{Haplotype networks of Echinococcus granulosus (s.s.)} Of the 83 E. granulosus (s.s.) isolates, 15 haplotypes resulted from the analysis of nad 1 gene sequences (Fig. 2a, Table 2) and 14 from nad5 gene (Fig. 2b, Table 2). The nad 1 and nad5 networks formed a star-like configuration with a central haplotype TB1 and bTB2, respectively, constituting $57.8 \%$ and $41 \%$, respectively, of the total E. gransuslosus (s.s.) population; these were separated from the remaining haplotypes by 1-3 (nad1) and 1-7 (nad5) mutational steps (Fig. 2a, b). The main haplotype in the nad 1 network was found in 3 out of 4 of the investigated counties (Zhongba, Saga and Dangxiong) while the nad5 main haplotype was found in 2 (Zhongba and Saga) of the counties. In addition, all six G3 isolates based on the $n a d 1$ gene formed a single haplotype (TB9) with three mutational steps from the founder haplotype (TB1) and haplotypes bTB12, bTB13 and bTB14 based on the nad5 gene had 5-7 mutation steps from the main haplotype (bTB2) (Fig. 2a, b).

The diversity and neutrality indices of E. granulosus (s.s.) were calculated based on the sequences of nad 1 and nad5 and indicated population expansion (Table 3). The pairwise fixation $\left(\mathrm{F}_{\mathrm{ST}}\right)$ value between isolates from both host populations was low ( $\operatorname{ad} 1=0.04925$; nad5 $=0.06644$ ) suggesting the lack of genetic differentiation.

Haplotypes representing nucleotide sequences of $E$. granulosus (s.s.) nad 1 and nad5 genes have been deposited in GenBank under accession numbers MN269986MN270000 (nad1) and MN270001-MN270014 (nad5).

\section{Echinococcus canadensis phylogeny and network analysis}

The E. canadensis (G6) was detected in a sheep and yak from Zhongba (isolated from the liver) and Angren (isolated from the lungs) counties, respectively based on an initial analysis of the nad 1 gene. Further BLAST query of the complete mitochondrial genome sequences $(13,731 \mathrm{bp})$ gave a highest percentage coverage and similarity of $100 \%$ and $99.86 \%$ with genotype G6 (GenBank: AB208063) and $100 \%$ and $99.58 \%$ with genotype G7
(GenBank: AB235847), respectively. The genotype status was further confirmed by a median-joining network constructed by the nad2-nad5 gene sequences which provided a clear distinction from reference $\mathrm{G} 7$ nucleotide sequences (Fig. 3). The Bayesian phylogeny based on the 12 protein coding regions showed the G6 isolates from this study in the same cluster with other G6 sequences (Additional file 4: Figure S3). The reference G6/G7 sequences used in this analysis were from Laurimae et al. [29]. The resulting mitochondrial genome sequences of E. canadensis (G6) from this study were submitted to the GenBank database under the accession numbers MN340038 (YakCHN1) and MN340039 (ShpCHN2).

\section{Discussion}

The Tibet Autonomous Region is one of the most CE endemic regions in the country. Previous reports have found prevalence to reach up to $10 \%$ in humans and $82 \%$ in intermediate hosts [30]. However, recent estimates have shown a huge decrease in the prevalence of $C E$ with an average of $1.66 \%$ in humans and $11.84 \%$ in livestock [19]. In the region, CE transmission is usually maintained by the pastoral lifestyle of the inhabitants including the use of guard dogs which play a significant role in the transmission pattern through faecal contamination of the environment.

To date, a number of investigations have shed light on the genetic diversity within the genus Echinococcus and the intraspecific variation among $E$. granulosus (s.s.) using different DNA markers $[1,2,31,32]$. In the present study, using mitochondrial DNA, the results lend support to previous observation of E. granulosus (s.s.) (G1) as the leading cause of $\mathrm{CE}$ in the Tibetan plateau indicating that of all species/genotypes of Echinococcus responsible for $\mathrm{CE}$, this highly zoonotic group predominates in China [7-12].

Overall, the genetic variation observed among E. granulosus (s.s.) isolates and the results of neutrality indices as supported by the significant negative values of both Tajima's $D$ and Fu's Fs indicated a negative selection or

Table 1 Number of isolates analysed from all four counties in the Tibet Autonomous Region of China (TAR)

\begin{tabular}{llll}
\hline Location & \multicolumn{2}{l}{ Isolate/host } & Total \\
\cline { 2 - 3 } & Sheep & Yak & \\
\hline Angren county & - & 1 & 1 \\
Dangxiong county & 1 & - & 1 \\
Saga county & 14 & 19 & 33 \\
Zhongba county & 39 & 11 & 50 \\
Total & 54 & 31 & 85 \\
\hline
\end{tabular}


$\mathbf{a}$
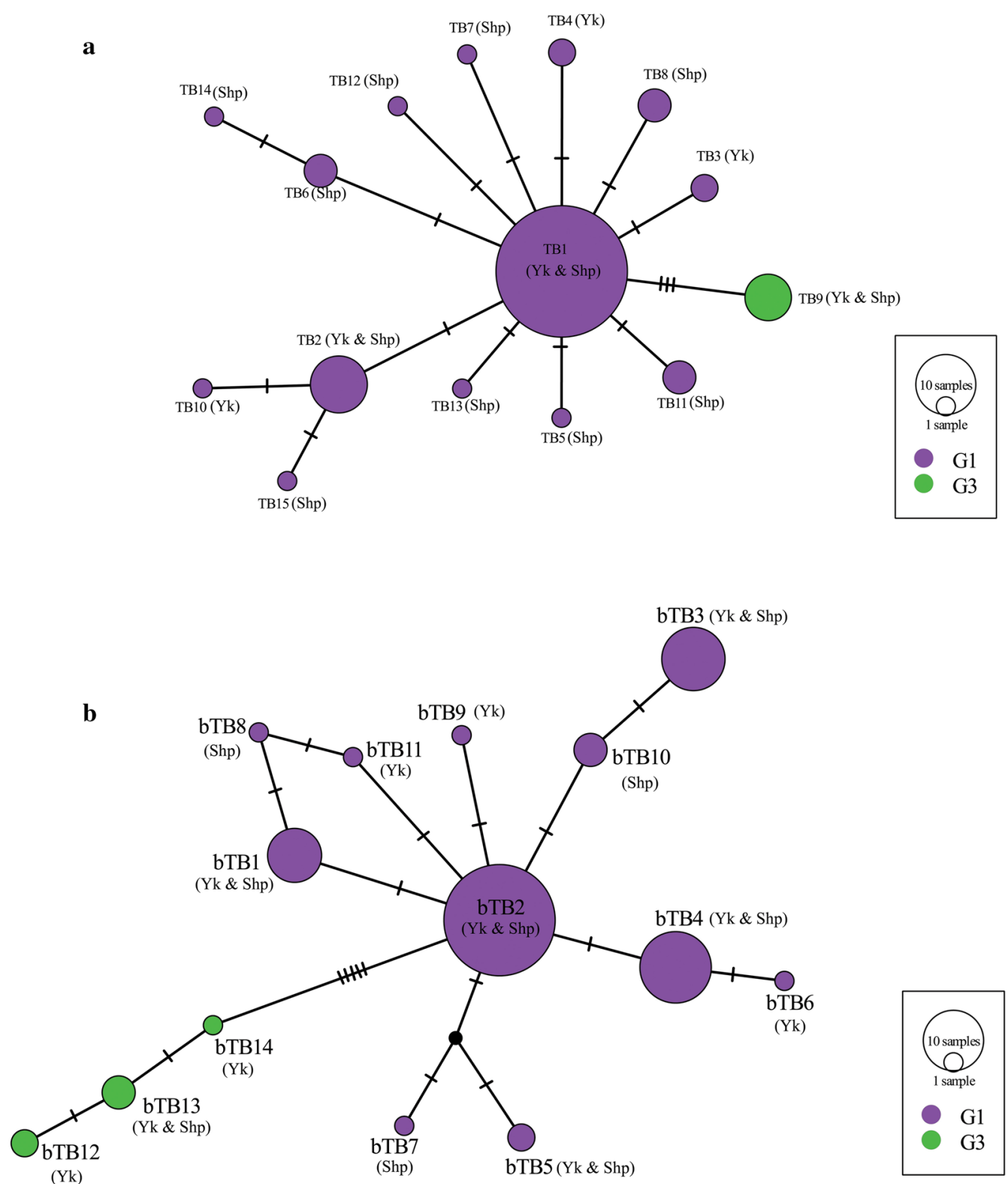

Fig. 2 Median-joining networks of Tibetan population of 83 Echinococcus granulosus (s.s.) isolates (yak, $n=30$, sheep, $n=53$ ) a nad1 (894 bp) and b nad5 (680 bp). Circle sizes are proportional to the corresponding haplotype frequencies. Hatch marks represent the number of mutations

population expansion, a common feature of Echinococcus spp. population in the Tibetan plateau [10, 13, 31]. The low nucleotide diversity among E. granulosus (s.s.) in the present study has also been previously observed in the Qinghai-Tibet region and other parts of China $[13,31]$ as well as from outside China [32, 33], supporting the demographical expansion of the group. Also, the low $\mathrm{F}_{\mathrm{ST}}$ value between the intermediate host populations suggests that both populations are genetically undifferentiated, indicating gene flow and alleles sharing between the subpopulations of E. granulosus (s.s.) and possibly with the overall population in the region which is plausible considering that both intermediate hosts utilized the same environment with no geographical barrier and are exposed to similar risk factors like contamination of pastures with faeces of infected dogs. The onset implication of gene flow on the parasite population includes increase genetic variation and host adaptability which over time, in the absence of natural selection and/or genetic drift, may reach gene homogeneity due to allele frequencies reaching equilibrium values and thus the appearance of a population structure characterised by a founder haplotype that is 
Table 2 Echinococcus granulosus (s.s.) haplotypes showing host, origin and their corresponding GenBank accession numbers

\begin{tabular}{|c|c|c|c|c|c|c|c|c|c|}
\hline \multicolumn{5}{|l|}{ nad1 } & \multicolumn{5}{|l|}{ nad5 } \\
\hline Haplotype & Host & Origin & Freq. & GenBank ID & Haplotype & Host & Origin & Freq. & GenBank ID \\
\hline TB1 & Sheep and Yak & Zhongba, Saga, Dangxiong & 48 & MN269986 & bTB1 & Sheep and Yak & Zhongba, Saga & 8 & MN270001 \\
\hline TB2 & Sheep and Yak & Zhongba, Saga & 9 & MN269987 & bTB2 & Sheep and Yak & Zhongba, Saga & 34 & MN270002 \\
\hline TB3 & Yak & Zhongba & 2 & MN269988 & bTB3 & Sheep and Yak & Zhongba, Dangxiong & 11 & MN270003 \\
\hline TB4 & Yak & Saga & 2 & MN269989 & bTB4 & Sheep and Yak & Zhongba, Saga & 14 & MN270004 \\
\hline TB5 & Sheep & Zhongba & 1 & MN269990 & bTB5 & Sheep and Yak & Zhongba & 2 & MN270005 \\
\hline TB6 & Sheep & Zhongba, Saga & 3 & MN269991 & bTB6 & Yak & Zhongba & 1 & MN270006 \\
\hline TB7 & Sheep & Saga & 1 & MN269992 & bTB7 & Sheep & Zhongba & 1 & MN270007 \\
\hline TB8 & Sheep & Zhongba, Saga & 3 & MN269993 & bTB8 & Sheep & Zhongba & 1 & MN270008 \\
\hline TB9 & Sheep and Yak & Zhongba, Saga & 6 & MN269994 & bTB9 & Yak & Saga & 1 & MN270009 \\
\hline TB10 & Yak & Zhongba & 1 & MN269995 & bTB10 & Sheep & Zhongba, Saga & 3 & MN270010 \\
\hline TB11 & Sheep & Zhongba & 3 & MN269996 & bTB11 & Yak & Saga & 1 & MN270011 \\
\hline TB12 & Sheep & Zhongba & 1 & MN269997 & bTB12 & Yak & Saga & 2 & MN270012 \\
\hline TB13 & Sheep & Saga & 1 & MN269998 & bTB13 & Sheep and Yak & Zhongba & 3 & MN270013 \\
\hline TB14 & Sheep & Saga & 1 & MN269999 & bTB14 & Yak & Zhongba & 1 & MN270014 \\
\hline TB15 & Sheep & Zhongba & 1 & MN270000 & & & & & \\
\hline
\end{tabular}

Table 3 Diversity and neutrality indices for Echinococcus granulosus (s.s.) populations from Tibet Autonomous Region of China (TAR)

\begin{tabular}{|c|c|c|c|c|c|c|}
\hline \multirow[t]{2}{*}{ Index } & \multicolumn{3}{|c|}{ nad1 (894 bp) } & \multicolumn{3}{|c|}{ nad5 (683 bp) } \\
\hline & Yak & Sheep & Overall & Yak & Sheep & Overall \\
\hline No. of isolates & 30 & 53 & 83 & 30 & 53 & 83 \\
\hline No. of mutations & 7 & 13 & 16 & 16 & 14 & 17 \\
\hline Parsimony informative site & 6 & 4 & 9 & 9 & 5 & 14 \\
\hline No. of haplotypes & 6 & 12 & 15 & 11 & 9 & 14 \\
\hline Haplotype diversity (Hd) & 0.618 & 0.663 & 0.650 & 0.818 & 0.755 & 0.782 \\
\hline Nucleotide diversity $(\pi)$ & 0.00154 & 0.00107 & 0.00127 & 0.00411 & 0.00230 & 0.00306 \\
\hline Tajima's D & -0.660 & $-1.973^{*}$ & $-1.851^{*}$ & -1.03778 & -1.475 & -1.119 \\
\hline Fu's Fs & -0.715 & $-8.472^{*}$ & $-10.098^{*}$ & -2.589 & -2.013 & -3.922 \\
\hline
\end{tabular}

* Significant $P$-value

common to different host populations as seen in the present study. Similar observations of gene flow and the lack of genetic differentiation among populations of E. granulosus (s.s.) has also been reported in the Qinghai-Tibet region $[10,13,31,34]$ and other locations [32, 35].

The main haplotype of E. granulosus (s.s.), TB1, based on the nad 1 network showed a $100 \%$ homology to Tunisian, Spanish, Turkish and Chinese isolates from sheep, Chilean isolate from cattle, and human isolates from Algeria and Finland [10,36]. Similarly, the nad5 main haplotype also showed 100\% similarity to GenBank sequences including Australian G1 isolate from sheep [37], Tunisian and Algerian human G1 isolates and Chilean cattle isolates [36]. This observation supports the diffusion theory of E. granulosus (s.s.) group from the Middle East where it is believed to have originated as a result of sheep domestication and the subsequent dispersal of the tapeworm to Europe, Africa, the Americas and Asia by the anthropogenic movement of intermediate host animals through livestock trade [12, 38].

Differences in genetic variation as reflected in haplotype distribution among different populations of $E$. granulosus (s.s.) in the Tibetan Plateau and other parts of China have also been observed [11, 31]. However, these differences could be a result of the short gene sequences analysed [39], the mitochondrial region investigated or the dissimilarities in the evolution rate of the various mitochondrial genes [13]. For instance, 83 isolates in this study, resulted in 15 haplotypes based on the complete mitochondrial nad 1 gene while the partial nad5 gene resulted in 14 and are incomparable to the 28 haplotypes from 84 E. granulosus (s.s.) 


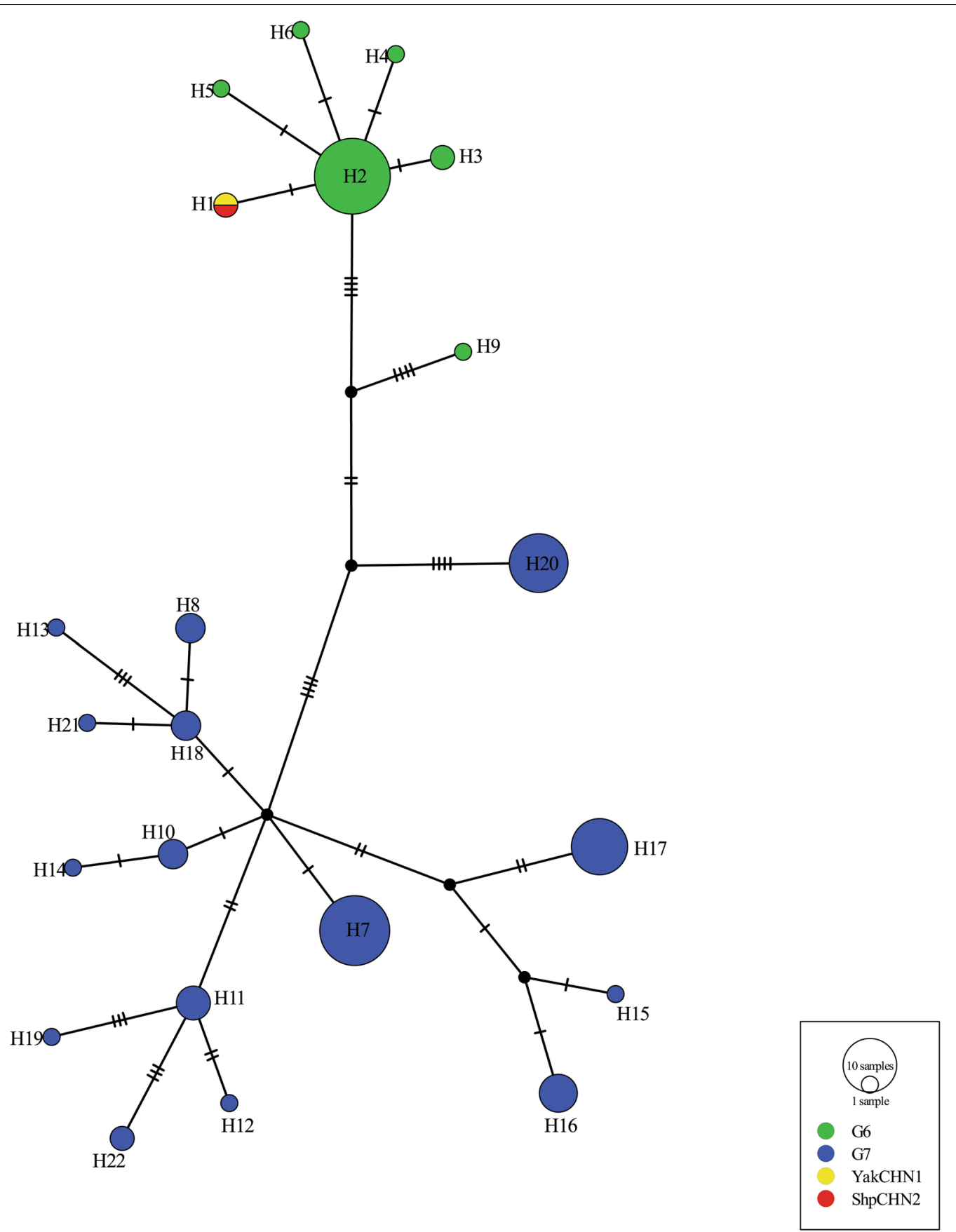

Fig. 3 Median-joining network of concatenated nad2-nad5 (2454 bp) genes of Tibetan Echinococcus canadensis G6 isolates. Hatch marks represent the number of mutations. E. canadensis G6/G7 GenBank reference sequences MH300930-MH300954, MH300971 (Gmon) and MH300956-MH300970, MH300972-MH301022 (G7) were obtained from Laurimae et al. [28]. H9 represent the Gmon isolate from Mongolia whose phylogenetic relationship with the $\mathrm{G} 6$ and $\mathrm{G} 7$ genotypes and genetic identity remain unclear

isolates from the Tibetan Plateau based on a concatenated sequence of nad 1 and atp6 genes [10], 19 haplotypes reported by Wang et al. [13], using mitochondrial nad 2 gene as well as 10 haplotypes among 45 isolates from humans, sheep, and yaks in western China based on mitochondrial cytb gene [11]. Nonetheless, these reports support the existence of intraspecific variation within E. granulosus (s.s.) and an expanding population in the region.

The population network of E. granulosus (s.s.) in this region as depicted by the median-joining network was characterised by a star-like configuration with a centrally 
placed haplotype similar to previous observations $[12,13$, 31]. Meanwhile, a study observed that the phylogenetic network of E. granulosus (s.s.) in the Tibetan region [12] differs considerably with that of the Middle East $[39,40]$ where E. granulosus (s.s.) is also prevalent [32, 39, 41] such that the Tibetan phylogenetic network is characteristically star-shaped with a centrally placed haplotype and low genetic variation in contrast to that of the Middle East but was found to be somewhat similar to the radial phylogenetic network reported in Africa [32]. This pattern of network is also characteristic of an expanding population and again strengthens the diffusion theory of the group from the Middle East. Conversely, recent studies based on the analysis of near-complete mitochondrial genomes of a large dataset of E. granulosus (s.s.) isolates from different hosts/regions suggested the absence of a central haplotype [36, 42]. However, the observation of a central haplotype in the present study and in most studies could be a geographical limitation or a result of short DNA fragment analysed.

The $E$. canadensis G6 isolates from this study on comparison with the available partial gene sequences of $E$. canadensis G6 and G7 from the Tibetan Plateau region revealed some differences involving nucleotide base substitutions. For instance, a comparison of the partial nad 1 gene sequences of $E$. canadensis identified as G7 from goat in Qinghai (GenBank: JQ317986) with our nad1 gene sequences showed changes at position $477(\mathrm{C} / \mathrm{T})$. Similarly, changes at positions $717(\mathrm{~T} / \mathrm{C}), 723(\mathrm{~A} / \mathrm{T}), 1122$ $(\mathrm{A} / \mathrm{G})$ and $1125(\mathrm{~A} / \mathrm{G})$ were also observed with the partial cox 1 gene sequences of $E$. canadensis G6 genotype from a human in Xinjiang (GenBank: DQ356884). Furthermore, comparison of the complete cox 2 gene sequences from this study with the partial cox 2 gene sequences of the G6 genotype previously isolated from sheep in the Tibet Autonomous Region (GenBank: KC692991, KC692992) showed similar nucleotide sequences without variation. However, the G6 genotype from this study, confirmed by comparing the complete mitochondrial genome sequences with reference G6 and G7 genotypes available in GenBank and the phylogenetic network of the nad2-nad5 gene sequences [24], possessed the G6 peculiar nucleotide bases at the defining positions for E. granulosus (s.l.) genotypes G6 and G7 as previously described [24].

The presence of the highly zoonotic E. granulosus (s.s.) in China remains a serious public health concern as the country accounts for over $90 \%$ of the global human CE burden [43]. More so, the growing evidence of human and livestock infection with members of the $E$. canadensis group raises more public health concerns, suggesting the need for improved surveillance in order to appraise the level of infection due to members of this group. Although integrated measures which include control of the source of infection by deworming of dogs with anthelmintics, strict management practices in slaughterhouses and quarantine of livestock to restrict dogs from feeding on offal of infected animals, health education on the need for improved personal hygiene, and treatment of patients, are in place to mitigate $\mathrm{CE}$ transmission and to achieve control. However, more still needs to be done in the area of surveillance and appraisal of the control efforts in order to achieve control/eradication. Also, in the event of the use of vaccines, it is important to understand the species/genetic diversity of Echinococcus in an endemic area as immune response may differ due to infection by different genotypes which consequently affect control efforts. For example, the EG95 orthologue in E. canadensis and E. granulosus (G1) has been found to differ in their amino acid constitution which could affect their immunogenic properties [44].

\section{Conclusions}

Although E. canadensis G6 genotype has been reported in sheep from the Tibet Autonomous Region, to the best of our knowledge, this is the first report to describe detection of this genotype in a yak, suggesting the role of sheep and yaks in maintaining CE infection caused by the G6 genotype. This highlights the need to consider the role of other livestock in the transmission and maintenance of the G6 genotype and the contributions to human infection during control programmes. The study also provided additional data on the genetic variation of E. granulosus (s.s.) in the Tibet Autonomous Region.

\section{Supplementary information}

Supplementary information accompanies this paper at https://doi. org/10.1186/s13071-019-3857-1.

Additional file 1: Figure S1. Sample images of infected lungs (a) and liver (b) of sheep.

Additional file 2: Figure S2. Microscopic images of protoscoleces from hydatid cysts $100 \times$ magnification (a) and $400 \times$ magnification (b).

Additional file 3: Table S1. List of overlapping primers used in amplifying the complete Echinococcus canadensis G6 mitochondrial genome. Table S2. Mutation sites of nad1 and nad5 genes of Echinococcus granulosus (s.s.) haplotypes found in the Tibet Autonomous Region of China (TAR). Table S3. Amino acid changes resulting from nad 1 and nad5 nucleotide mutations.

Additional file 4: Figure S3. Bayesian phylogeny of Echinococcus canadensis (G6) isolates from Tibet and other G6 (GenBank: MH300930$\mathrm{MH} 300954, \mathrm{MH} 300971)$ and G7 (MH300956-MH300970, MH300972MH301022) sequences from different countries retrieved from GenBank based on the 12 protein-coding mitochondrial genes. Red indicates isolates from this study [GenBank: MN340038 (YakCHN1) and MN340039 (ShpCHN2)]. Echinococcus canadensis G6/G7 reference sequences are from Laurimae et al. [29]. Note that the sequence with GenBank accession $\mathrm{MH} 300971$, is the "Gmon" isolate from Mongolia whose genotypic identity and phylogenetic relationship with the $\mathrm{G} 6$ and $\mathrm{G} 7$ genotypes remain unclear. 


\begin{abstract}
Abbreviations
PBS: phosphate buffer solution; PCR: polymerase chain reaction; cox1: cytochrome $c$ oxidase subunit 1 gene; nad1: NADH dehydrogenase subunit 1 gene; nad2: NADH dehydrogenase subunit 2 gene; nad5: NADH dehydrogenase subunit 5 gene; CE: cystic echinococcosis; MCMC: Markov Chain Monte Carlo; PSRF: potential scale reduction factor; $\mathrm{Hd}$ : haplotype diversity; atp6: adenosine triphosphatase subunit 6 gene; RNA: ribonucleic acid.
\end{abstract}

\section{Acknowledgements}

We would like to thank the abattoir staff in each county in the study area for their assistance during sample collection.

\section{Authors' contributions}

JAO, HBY and WZJ conceived and designed the experiments. CYX, JZL, WQT, $D$, and BS collected the samples. JAO, LL, HBY, YTW and GQZ, performed the experiments and the data analyses. JAO wrote the first draft of the manuscript, and $\mathrm{HBY}, \mathrm{BQF}, \mathrm{HBY}$ and WZJ provided comments and suggestions for revisions. All authors read and approved the final manuscript.

\section{Funding}

This study was supported by the Open fund of State Key Laboratory of Veterinary Etiological Biology (SKLVEB2016KFKT011), the Central Public-interest Scientific Institution Basal Research Fund (1610312017001; 1610312016012), the National Key Research and Development Plan (2017YFD0501301), and NBCITS (CARS-37). John Asekhaen Ohiolei's PhD scholarship was provided by the Chinese Government Scholarship (CGS) Programme, The Peoples Republic of China.

\section{Availability of data and materials}

All data supporting the conclusions of this article are included in the article and its additional files. Representative nad 1 and nad5 sequences of Echinococcus granulosus (s.s.) and the complete mitochondrial genome sequences of $E$. canadensis $\mathrm{G} 6$ from the present study were submitted to the GenBank database under the accession numbers MN269986-MN270014 and MN340038MN340039, respectively.

\section{Ethics approval and consent to participate}

Not applicable.

\section{Consent for publication}

Not applicable.

\section{Competing interests}

The authors declare that they have no competing interests.

\section{Author details}

${ }^{1}$ State Key Laboratory of Veterinary Etiological Biology/National Professional Laboratory of Animal Hydatidosis, Key Laboratory of Veterinary Parasitology of Gansu Province/Lanzhou Veterinary Research Institute, CAAS, Lanzhou 730046, Gansu, People's Republic of China. ${ }^{2}$ Institute of Animal Science of Tibet Academy of Agricultural and Animal Husbandry Sciences, Lhasa 854000, Tibet Autonomous Region, People's Republic of China.

Received: 23 August 2019 Accepted: 16 December 2019

Published online: 27 December 2019

\section{References}

1. Bowles J, Blair D, McManus D. Genetic variants within the genus Echinococcus identified by mitochondrial DNA sequencing. Mol Biochem Parasitol. 1992;54:165-73.

2. Bowles J, McManus DP. NADH dehydrogenase 1 gene sequences compared for species and strains of the genus Echinococcus. Int J Parasitol. 1993;23:969-72.

3. Lavikainen A, Lehtinen MJ, Meri T, Hirvelä-Koski V, Meri S. Molecular genetic characterization of the Fennoscandian cervid strain, a new genotypic group (G10) of Echinococcus granulosus. Parasitology. 2003;127:207-15.
4. Lymbery AJ. Phylogenetic pattern, evolutionary processes and species delimitation in the genus Echinococcus. Adv Parasitol. 2017;95:111-45.

5. Thompson R. The taxonomy, phylogeny and transmission of Echinococcus. Exp Parasitol. 2008;119:439-46.

6. Lymbery AJ, Jenkins EJ, Schurer JM, Thompson RC. Echinococcus canadensis, E. borealis, and E. intermedius. What's in a name? Trends Parasitol. 2015;31:23-9.

7. Yang YR, Rosenzvit MC, Zhang LH, Zhang JZ, McManus DP. Molecular study of Echinococcus in west-central China. Parasitology. 2005;131:547-55

8. Li T, Ito A, Nakaya K, Qiu J, Nakao M, Zhen R, et al. Species identification of human echinococcosis using histopathology and genotyping in northwestern China. Trans R Soc Med Hyg. 2008;102:585-90.

9. Ma SM, Maillard S, Zhao HL, Huang X, Wang H, Geng PL, et al. Assessment of Echinococcus granulosus polymorphism in Qinghai Province, People's Republic of China. Parasitol Res. 2008;102:1201-6.

10. Yan N, Nie HM, Jiang ZR, Yang AG, Deng SJ, Guo L, et al. Genetic variability of Echinococcus granulosus from the Tibetan plateau inferred by mitochondrial DNA sequences. Vet Parasitol. 2013;196:179-83.

11. Zhong X, Wang N, Hu D, Wang J, Liu T, Gu X, et al. Sequence analysis of cytb gene in Echinococcus granulosus from western China. Korean J Parasitol. 2014;52:205-9.

12. Hu D, Song X, Wang N, Zhong X, Wang J, Liu T, et al. Molecular identification of Echinococcus granulosus on the Tibetan Plateau using mitochondrial DNA markers. Genet Mol Res. 2015;14:13915-23.

13. Wang J, Wang N, Hu D, Zhong X, Wang S, Gu X, et al. Genetic diversity of Echinococcus granulosus in southwest China determined by the mitochondrial NADH dehydrogenase subunit 2 gene. Sci World J. 2014:867839.

14. Zhang T, Yang D, Zeng Z, Zhao W, Liu A, Piao D, et al. Genetic characterization of human-derived hydatid cysts of Echinococcus granulosus sensu lato in Heilongjiang Province and the first report of G7 genotype of $E$. canadensis in humans in China. PLoS ONE. 2014;9:e109059.

15. Wu Y, Li L, Zhu G, Li W, Zhang N, Li S, et al. Mitochondrial genome data confirm that yaks can serve as the intermediate host of Echinococcus canadensis (G10) on the Tibetan Plateau. Parasites Vectors. 2018;11:166.

16. Zhang LH, Chai JJ, Jiao W, Osman Y, McManus DP. Mitochondrial genomic markers confirm the presence of the camel strain (G6 genotype) of Echinococcus granulosus in north-western China. Parasitology. 1998;116:29-33.

17. Bart JM, Abdukader M, Zhang YL, Lin RY, Wang YH, Nakao M, et al. Genotyping of human cystic echinococcosis in Xinjiang, PR China. Parasitology. 2006;133:571-9.

18. Liu Q, Cao L, Zhang Y, Xu D, Shang L, Wang X, et al. Genotypes of Echinococcus granulosus in animals from Yushu, northeastern China. Vector Borne Zoonotic Dis. 2013;13:134-7.

19. Li B, Quzhen G, Xue CZ, Han S, Chen WQ, et al. Epidemiological survey of echinococcosis in Tibet Autonomous Region of China. Infect Dis Poverty. 2019;8:29.

20. Kinkar L, Laurimäe T, Acosta-Jamett G, Andresiuk V, Balkaya I, Casulli A, et al. Distinguishing Echinococcus granulosus sensu stricto genotypes G1 and G3 with confidence: a practical guide. Infect Genet Evol. 2018;64:178-84.

21. Hall TA. BioEdit: a user-friendly biological sequence alignment editor and analysis program for Windows 95/98/NT. Nucl Acids Symp Ser. 1999;41:95-8.

22. Tillich M, Lehwark P, Pellizzer T, Ulbricht-Jones ES, Fischer A, Bock R, Greiner S. GeSeq - versatile and accurate annotation of organelle genomes. Nucl Acids Res. 2017;45:W6-11.

23. Rozas J, Ferrer-Mata A, Sanchez-DelBarrio JC, Guirao Librado P, RamosOnsins SE, Sanchez-Gracia A. DnaSP v6: DNA sequence polymorphism analysis of large datasets. Mol Biol Evol. 2017;34:3299-302.

24. Laurimäe T, Kinkar L, Romig T, Umhang G, Casulli A, Omer RA, et al. Analysis of nad 2 and nad5 enables reliable identification of genotypes $\mathrm{G} 6$ and G7 within the species complex Echinococcus granulosus sensu lato. Infect Genet Evol. 2019;74:103941.

25. Leigh JW, Bryant D. PopART: full-feature software for haplotype network construction. Methods Ecol Evol. 2015;6:1110-6. 
26. Tajima F. Statistical method for testing the neutral mutation hypothesis by DNA polymorphism. Genetics. 1989;123:585-95.

27. Fu YX. Statistical tests of neutrality of mutations against population growth, hitchhiking and background selection. Genetics. 1997; 147:915-25.

28. Gelman A, Rubin DA. Inference from iterative simulation using multiple sequences. Stat Sci. 1992;7:457-72.

29. Laurimae T, Kinkar L, Romig T, Omer RA, Casulli A, Umhang G, et al. The benefits of analysing complete mitochondrial genomes: deep insights into the phylogeny and population structure of Echinococcus granulosus sensu lato genotypes G6 and G7. Infect Genet Evol. 2018;64:85-94.

30. Zhang W, Zhang Z, Wu W, Shi B, Li J, Zhou X, et al. Epidemiology and control of echinococcosis in central Asia, with particular reference to the People's Republic of China. Acta Trop. 2015;141:235-43.

31. Nakao M, Li T, Han X, Ma X, Xiao N, Qiu J, et al. Genetic polymorphisms of Echinococcus tapeworms in China as determined by mitochondrial and nuclear DNA sequences. Int J Parasitol. 2010;40:379-85.

32. Boufana B, Lahmar S, Rebaï W, Ben Safta Z, Jebabli L, Ammar A, et al. Genetic variability and haplotypes of Echinococcus isolates from Tunisia. Trans R Soc Trop Med Hyg. 2014;108:706-14.

33. Alvarez Rojas CA, Ebi D, Paredes R, Acosta-Jamett G, Urriola N, Roa JC, et al. High intraspecific variability of Echinococcus granulosus sensu stricto in Chile. Parasitol Int. 2017;66:112-5.

34. Wang N, Wang J, Hu D, Zhong X, Jiang Z, Yang A, et al. Genetic variability of Echinococcus granulosus based on the mitochondrial 165 ribosomal RNA gene. Mitochondrial DNA. 2015;26:396-401.

35. Casulli A, Interisano M, Sreter T, Chitimia L, Kirkova Z, La Rosa G, et al. Genetic variability of Echinococcus granulosus sensu stricto in Europe inferred by mitochondrial DNA sequences. Infect Genet Evol. 2012;12:377-83.

36. Kinkar L, Laurimae T, Acosta-Jamett G, Andresiuk V, Balkaya I, Casulli A, et al. Global phylogeography and genetic diversity of the zoonotic tapeworm Echinococcus granulosus sensu stricto genotype G1. Int J Parasitol. 2018;48:729-42.
37. Kinkar L, Korhonen PK, Cai H, Gauci CG, Lightowlers MW, Saarma U, et al. Long-read sequencing reveals a $4.4 \mathrm{~kb}$ tandem repeat region in the mitogenome of Echinococcus granulosus (sensu stricto) genotype G1. Parasites Vectors. 2019;12:238.

38. Yanagida T, Mohammadzadeh T, Kamhawi S, Nakao M, Sadjjadi SM, Hijjawi N, et al. Genetic polymorphisms of Echinococcus granulosus sensu stricto in the Middle East. Parasitol Int. 2012;61:599-603.

39. Jafari R, Sanei B, Baradaran A, Spotin A, Bagherpour B, Darani H. Genetic characterization of Echinococcus granulosus strains isolated from humans based on nad1 and cox1 gene analysis in Isfahan, central Iran. J Helminthol. 2018;92:696-702.

40. Spotin A, Mahami-Oskouei M, Harandi MF, Baratchian M, Bordbar A, Ahmadpour E, et al. Genetic variability of Echinococcus granulosus complex in various geographical populations of Iran inferred by mitochondrial DNA sequences. Acta Trop. 2017;165:10-6.

41. Mulinge E, Magambo J, Odongo D, Njenga S, Zeyhle E, Mbae C, et al. Molecular characterization of Echinococcus species in dogs from four regions of Kenya. Vet Parasitol. 2018;255:49-57.

42. Kinkar L, Laurimäe T, Balkaya I, Casulli A, Zait H, Irshadullah M, et al. Genetic diversity and phylogeography of the elusive, but epidemiologically important Echinococcus granulosus sensu stricto genotype G3. Parasitology. 2018;145:1613-22.

43. Deplazes P, Rinaldi L, Alvarez Rojas CA, Torgerson PR, Harandi MF, Romig T, et al. Global distribution of alveolar and cystic echinococcosis. Adv Parasitol. 2017;95:315-493.

44. Chow C, Gauci CG, Vural G, Jenkins DJ, Heath DD, Rosenzvit MC, Harandi MF, Lightowlers MW. Echinococcus granulosus: variability of the hostprotective EG95 vaccine antigen in $\mathrm{G} 6$ and $\mathrm{G} 7$ genotypic variants. Exp Parasitol. 2008;119:499-505.

\section{Publisher's Note}

Springer Nature remains neutral with regard to jurisdictional claims in published maps and institutional affiliations.
Ready to submit your research? Choose BMC and benefit from:

- fast, convenient online submission

- thorough peer review by experienced researchers in your field

- rapid publication on acceptance

- support for research data, including large and complex data types

- gold Open Access which fosters wider collaboration and increased citations

- maximum visibility for your research: over $100 \mathrm{M}$ website views per year

At BMC, research is always in progress.

Learn more biomedcentral.com/submissions 